\title{
Overcoming immune system evasion by personalized immunotherapy
}

"...cancer's ability to induce tumor tolerance is incredibly complex ... Future efforts should focus on early detection as well as molecular and genetic/epigenetic characterization of the tumors."

Keywords: checkpoint blockade $\bullet$ epigenetics $\bullet$ immune evasion $\bullet$ immunotherapy $\bullet$ PD-1 - PD-L1

With the US FDA's approval of the first cancer vaccine for prostate cancer [1] and immune checkpoint blockade therapy against CTLA-4 for melanoma [2], the complex interaction between antitumor immune responses and tumor immune evasion is being intensely scrutinized. Despite a number of exciting preclinical studies and clinical research, most novel immunotherapies have demonstrated limited efficacy in early-phase clinical trials. The reason for this is multifactorial. First, novel agents have generally been tested in patients with treatment refractory, metastatic and large tumor burdens who failed multiple therapeutic regimens in the past. Second, cancer is an extremely heterogeneous disease, even within the same subtype. Single agent therapy, regardless of its type, will most likely be ineffective. Moreover, and probably most importantly, tumor cells have a large arsenal of adaptive capabilities that render targeted therapies ineffective.

\section{Personalized evasion mechanisms}

Immune system surveillance is a critical component that suppresses tumor cell propagation by tumor cell lysis or directly inhibiting its ability to grow or metastasize. However, the immune system can facilitate tumor growth by selecting for cells more likely to survive and conditioning the tumor microenvironment for tumor survival and outgrowth [3]. This process, termed immune editing, places a strong selection pressure on the tumors and is described in three phases: elimination, equilibrium and escape. During the elimination phase, natural killer cells and dendritic cells, which travel to nearby lymph nodes and activate tumor-specific $\mathrm{CD}^{+}{ }^{+}$and $\mathrm{CD}^{+} \mathrm{T}$ cells, work in conjunction to eliminate nascent tumors [3]. Subsequently, tumor cells are eliminated via activated cytotoxic $\mathrm{T}$ cells during the equilibrium phase. During this phase, however, resistant tumor cells are selected out by evading immune system detection depending on the mutations acquired during carcinogenesis [3]. The equilibrium phase continues as long as the immune system is able to eliminate tumor cells. Eventually, this process is exhausted. The remaining tumor cells evade immune system detection due to alterations in cell death pathways, acquisition of stem cell-like phenotypes, epigenetic transformation as well as downregulated antigen presentation [4].

Importantly, genetically unstable tumor cells are able to circumvent the selective pressure of immune surveillance and proceed to the escape phase. However, increased mutations within a tumor may also lead to expression of neoantigens rendering them more susceptible to targeted immunotherapies. For example, microsatellite unstable colon cancer tumors have been associated with increased infiltrating T-cell populations and a better prognosis compared with microsatellite stable colon cancer secondary to increased neoantigens as a result of defects in DNA mismatch repair genes [5]. Antigen-specific vaccines targeting known tumor antigens have been tested in multiple clinical trials but with limited success [6]. Ascertaining which tumor antigens are abundantly expressed and critical to tumor disease progression is an

\author{
Kevin C Soares ${ }^{1,2,3,4}$, \\ Lei Zheng ${ }^{1,2,3,4,5}$ \\ \& Nita Ahuja*,1,2,3,4,5,6
}

'Department of Surgery, Johns Hopkins University School of Medicine, Baltimore, MD, USA

${ }^{2}$ The Sidney Kimmel Cancer Center, Johns Hopkins University School of Medicine, Baltimore, MD, USA

${ }^{3}$ The Skip Viragh Center for Pancreatic Cancer Research and Clinical Care, Johns Hopkins University School of Medicine,

Baltimore, MD, USA

4The Sol Goldman Pancreatic Cancer Center, Johns Hopkins University School of Medicine, Baltimore, MD, USA ${ }^{5}$ Department of Oncology, Johns Hopkins University School of Medicine, Baltimore, MD, USA

${ }^{6}$ Department of Urology, Johns Hopkins University School of Medicine, Baltimore, MD, USA

*Author for correspondence:

Tel.: +1 4105026135

Fax: +1 4105020987

nahuja1@jhmi.edu 
important component of personalized vaccine therapy. Genetic characterization of tumor cells and identification of these neoantigens allows for the development of individualized mutagen-specific vaccines.

Our group has recently reported novel intratumoral lymphoid aggregates in pancreatic tumors after neoadjuvant whole-cell allogeneic GM-CSF vaccine therapy [7]. The genetic characterization of these tertiary lymphoid aggregates demonstrated upregulation of immunosuppressive regulatory immune mechanisms including PD-1, PD-L1 and the regulatory T-cell pathway [7]. Our preclinical data indicates that the combination of vaccine therapy with PD-1 or PD-L1 blockade in pancreatic cancer improves vaccine therapy by facilitating effector T-cell infiltration into the tumor microenvironment [SoAres KC ET AL., Unpublished Data]. As a result, gene signatures from these lymphoid aggregates can potentially be used to direct vaccine therapy with a personalized immune modulator.

\section{Epigenetic therapy \& its implication in personalized immunotherapy}

Understanding the genetic and epigenetic components of tumor immune evasion is critical. Most of the discussion regarding tumor immune evasion has centered on mutations in the cancer genome. For example, structural alterations in the MHC class I antigen-processing pathway allow for the immune escape of melanoma and microsatellite unstable colorectal cancer $[8,9]$. Notably, however, very few cancer genome mutations have been associated with a direct effect or correlated with immune evasion [10]. More recently, intense investigation on the relationship of epigenetic modulation and immune escape has revealed a dynamic and critical interplay between epigenetic and immune modulation, laying the groundwork for highly promising combinatorial therapies.

Epigenetic regulation of immunity includes direct regulation of genes involved in B-cell maturation, cytokine gene expression (i.e., IFN- $\gamma, I L-12, I L-10$ ), MHC class I and II expression and costimulatory genes (CD40, CD80, CD86) [11-13]. Certain tumors produce consistent immune inhibitory changes, such as in breast cancer where upregulation of immunosuppressive pathways including IL-10, TGF- $\beta$ and B7-H4 and downregulation of the T-cell costimulatory molecules CD28 and CD83 is commonly seen [4,14]. By contrast, other neoplasms produce markedly different immune signatures from one tumor to the next [14]. Therefore, there is a role for the determination of tumor immune signatures to more accurately tailor immunotherapeutics.

The tumor's immune suppressive pathways are dynamically related to epigenetic regulation and epigenetic therapy can specifically target this pathway in human cancers. In recent reports by our group, we demonstrated that low doses of DNA methyltransferase inhibitor 5-azacitidine (5-AZA) administered in vitro to human breast, ovarian, lung and colon cancer cell lines resulted in upregulation of immunomodulatory pathways, including IFN- $\gamma$, antigen-processing pathways, and cytokine and chemokine expression [15]. Moreover, correlative in vivo studies demonstrated similar findings [15]. In addition, upregulation of cancer antigens was also observed, specifically cancer testis antigen. Epigenetic modifications to antigen expression and presentation will vary among individual patients. Characterization of the response to epigenetic therapy in individual patients will lead to personalized therapies against specific tumor subtypes. For example, robust induction of cancer testis antigens in a subset of patients as a result of epigenetic therapy can be used as targets in antigen-specific vaccines and antibody therapy.

\section{"Future oncologic clinical investigations call for an ever-expanding role of personalized combinatorial therapies including chemotherapy, radiation, surgery, epigenetic modulation, immunotherapy and genetic characterization."}

Currently, clinical trials using epigenetic agents such as histone deacetylase inhibitors and demethylating agents (i.e., 5-AZA) focus directly on the therapeutic potential of these compounds. Although some studies have reported encouraging results in this regard [16-18], most have failed to demonstrate clinical efficacy as single agents in early phase clinical trials and recent reports suggest that an intact immune system is critical to the anticancer activities of these agents [19]. Therefore, as we gain a better understanding of epigenetics' role in immune system regulation, a new and important therapeutic role for epigenetic agents as part of combinatorial therapy is readily apparent.

Combination epigenetic therapy of 5-AZA and entinostat in patients with refractory advanced non-smallcell lung cancer patients demonstrated objective durable responses in only two of 65 patients according to response evaluation criteria in solid tumors (RECIST). After demonstrating disease progression, the remainder of these patients underwent a variety of treatment modalities including chemotherapy and immunotherapy targeting the immune checkpoint programmed death-1 (PD-1). When anti-PD-1 blockade was used in patients who had previously been primed with epigenetic therapy, most patients responded to antiPD-1 monotherapy [17,20]. Preclinical studies using an in vitro model characterized the 5-AZA-induced expression signature of immune genes and pathways, 
which are characteristically downregulated during immune surveillance, including the core interferon pathway transcription factor IRF7 [20]. The interplay of PD-1/PD-L1 interaction and epigenetic therapy reflects the complex interactions and synergistic possibilities. Epigenetic therapy targeting the tumor results in an increased proinflammatory response and reexpression of hypermethylated antigens. Concurrently, epigenetic therapy acts directly on $\mathrm{T}$ cells to increase IFN- $\gamma$ secretion as well as upregulate PD- 1 expression [21]. In addition, the elevated IFN- $\gamma$ levels upregulate tumor cell PD-L1 expression thereby abrogating this epigenetically induced immune activation.

Although some neoplasms are associated with optimal survival rates using current therapies, most tumors are poorly susceptible to current treatment modalities and new therapies are sorely needed. In addition to new therapies, a better understanding of the disease biology and its capability to render antineoplastic agents ineffective, even in the setting of initial clinical efficacy, argues for a different approach in addition to new treatment modalities. Cancer cells within individual primary tumors contain a variety of genetic, epigenetic and molecular variations. Therefore it is highly unlikely that a single antineoplastic agent will cure a given disease and history has thus far proven this to be true. Future oncologic clinical investigations call for an ever-expanding role of personalized combinatorial therapies including chemotherapy, radiation, surgery, epigenetic modulation, immunotherapy and genetic characterization. The cancer genome is constantly changing, therefore genetic and epigenetic biomarkerdriven combinatorial therapy will have the highest likelihood of success.

A core component of effective combinatorial, personalized therapy will be epigenetic therapy given that it is intricately related to our other treatment modalities. For example, chemotherapeutic agents induce epigenetic modifications in the epigenome. Alternatively, epigenetic therapy can render certain tumors sensitive

\section{References}

1 Kantoff PW, Higano CS, Shore ND et al. Sipuleucel-T immunotherapy for castration-resistant prostate cancer. N. Engl. J. Med. 363, 411-422 (2010).

2 Hodi FS, O'Day SJ, McDermott DF et al. Improved survival with ipilimumab in patients with metastatic melanoma. N. Engl. J. Med. 363, 711-723 (2010).

3 Schreiber RD, Old LJ, Smyth MJ. Cancer immunoediting: integrating immunity's roles in cancer suppression and promotion. Science 331, 1565-1570 (2011).

4 Bhatia A, Kumar Y. Cellular and molecular mechanisms in cancer immune escape: a comprehensive review. Expert Rev. Clin. Immunol. 10, 41-62 (2014). to a previously resistant chemotherapy regimen. Current ongoing trials at our institution are testing this premise (NCT01896856; NCT01935947). From an immunotherapeutic perspective, it is well established that epigenetic therapy upregulates previously suppressed immune effector genes as well as the expression of certain tumor antigens. Despite these promising results, future obstacles include identifying the appropriate strategy of combining therapies and identifying appropriate patient populations for those regimens.

\section{Conclusion}

In conclusion, cancer's ability to induce tumor tolerance is incredibly complex. Despite promising preclinical studies, durable clinical responses using novel immunotherapeutic agents are lacking. The complexity of cancer care is increasing daily. Future efforts should focus on early detection as well as molecular and genetic/epigenetic characterization of the tumors. Ultimately, these efforts will lead to early diagnosis and personalized, biomarker-driven combinatorial therapies using a variety of medical disciplines.

\section{Financial \& competing interests disclosure}

This work was supported by grants from the Americas Hepato-Pancreato-Biliary Association (AHPBA) (KC Soares), the Sol Goldman Pancreatic Cancer Center grants (KC Soares and L Zheng), NIH NIDDK T32 DK 7713-18 (KC Soares), National Cancer Institute (NCI) K23 CA127141 (N Ahuja), the American College of Surgeons/Society of University Surgeons (N Ahuja) and the Stand Up To Cancer (SU2C) Epigenetic Dream Team ( $N$ Ahuja). The authors have no other relevant affiliations or financial involvement with any organization or entity with a financial interest in or financial conflict with the subject matter or materials discussed in the manuscript. This includes employment, consultancies, honoraria, stock ownership or options, expert testimony, grants or patents received or pending or royalties.

No writing assistance was utilized in the production of this manuscript.

5 Pino MS, Chung DC. Microsatellite instability in the management of colorectal cancer. Expert Rev. Gastroenterol. Hepatol. 5, 385-399 (2011).

6 Gilliam AD, Broome P, Topuzov EG et al. An international multicenter randomized controlled trial of G17DT in patients with pancreatic cancer. Pancreas 41, 374-379 (2012).

7 Lutz ER, Wu AA, Bigelow E et al. Immunotherapy converts non-immunogenic pancreatic tumors into immunogenic foci of immune regulation. Cancer Immunol. Res. (2014) (In Press).

8 Bernal M, Ruiz-Cabello F, Concha A, Paschen A, Garrido F. Implication of the beta2-microglobulin gene in the generation of tumor escape phenotypes. Cancer Immunol. Immunother. 61, 1359-1371 (2012). 
9 Seliger B, Ritz U, Abele R et al. Immune escape of melanoma: first evidence of structural alterations in two distinct components of the MHC class I antigen processing pathway. Cancer Res. 61, 8647-8650 (2001).

10 Koscielny S. Why most gene expression signatures of tumors have not been useful in the clinic. Sci. Transl. Med. 2, $14 \mathrm{ps} 12$ (2010).

11 Tomasi TB, Magner WJ, Khan AN. Epigenetic regulation of immune escape genes in cancer. Cancer Immunol. Immunother. 55, 1159-1184 (2006).

12 Fonsatti E, Nicolay HJ, Sigalotti L et al. Functional upregulation of human leukocyte antigen class I antigens expression by 5 -aza-2'-deoxycytidine in cutaneous melanoma: immunotherapeutic implications. Clin. Cancer Res. 13, 3333-3338 (2007).

13 Simova J, Pollakova V, Indrova $\mathrm{M}$ et al. Immunotherapy augments the effect of 5-azacytidine on HPV16-associated tumours with different MHC class I-expression status. Br. J. Cancer 105, 1533-1541 (2011).

14 Sadun RE, Sachsman SM, Chen X et al. Immune signatures of murine and human cancers reveal unique mechanisms of tumor escape and new targets for cancer immunotherapy. Clin. Cancer Res. 13, 4016-4025 (2007).
15 Li H, Chiappinelli KB, Guzzetta AA et al. Immune regulation by low doses of the DNA methyltransferase inhibitor 5 -azacitidine in common human epithelial cancers. Oncotarget 5, 587-598 (2014).

16 Kaminskas E, Farrell A, Abraham S et al. Approval summary: azacitidine for treatment of myelodysplastic syndrome subtypes. Clin. Cancer Res. 11, 3604-3608 (2005).

17 Juergens RA, Wrangle J, Vendetti FP et al. Combination epigenetic therapy has efficacy in patients with refractory advanced non-small cell lung cancer. Cancer Discov. 1, 598-607 (2011).

18 Matei D, Fang F, Shen C et al. Epigenetic resensitization to platinum in ovarian cancer. Cancer Res. 72, 2197-2205 (2012).

19 West AC, Mattarollo SR, Shortt J et al. An intact immune system is required for the anticancer activities of histone deacetylase inhibitors. Cancer Res. 73, 7265-7276 (2013).

20 Wrangle J, Wang W, Koch A et al. Alterations of immune response of non-small cell lung cancer with azacytidine. Oncotarget 4, 2067-2079 (2013).

21 Zhang M, Xiao XQ, Jiang YF et al. DNA demethylation in PD-1 gene promoter induced by 5 -azacytidine activates PD-1 expression on Molt-4 cells. Cell Immunol. 271, 450-454 (2011). 